\title{
Dossier
}

El activismo urbano frente a la crisis socioambiental y climática

The urban activism against the socio-environmental and climatic crisis

Tommaso Gravante; Alice Poma, coords.

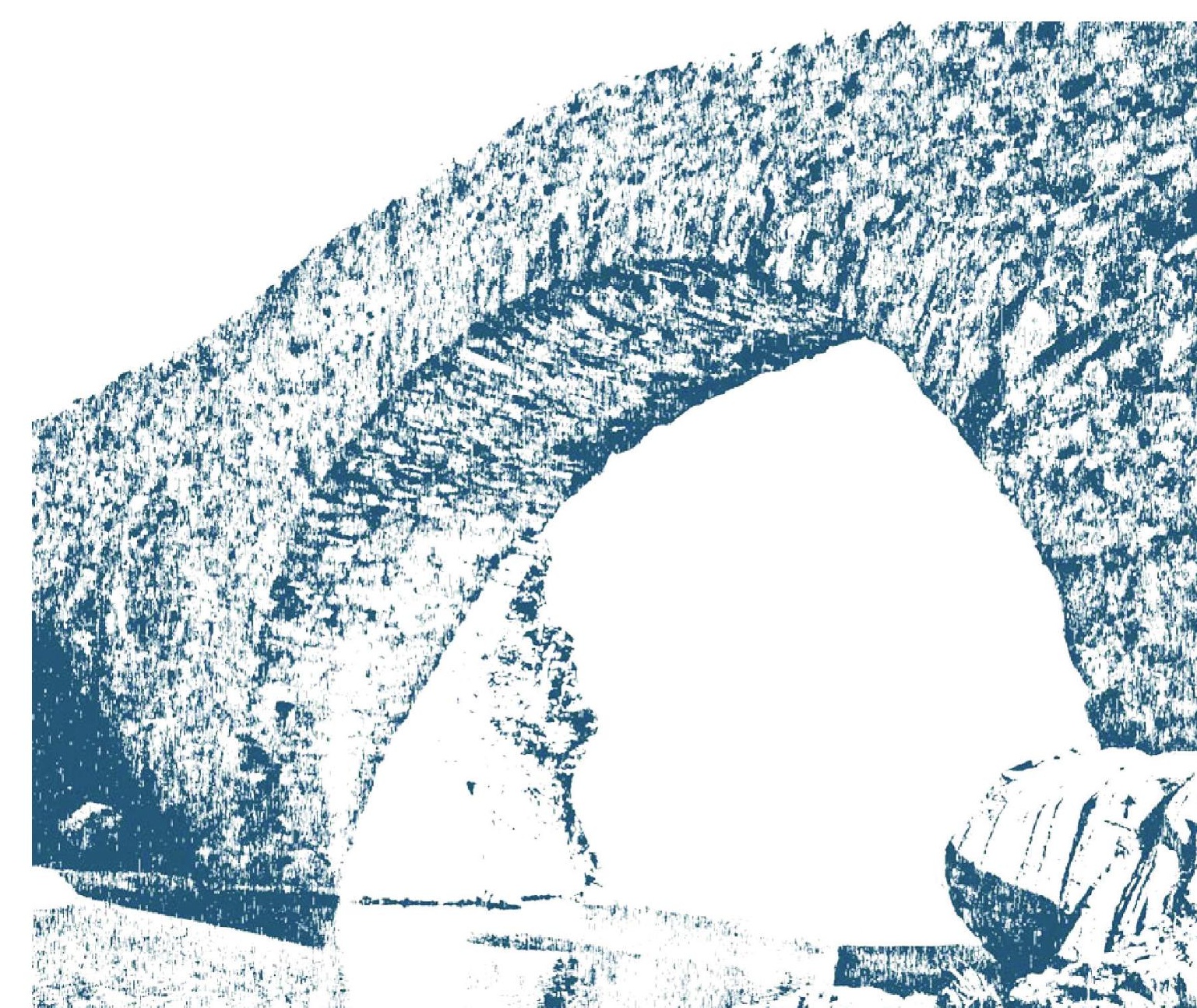





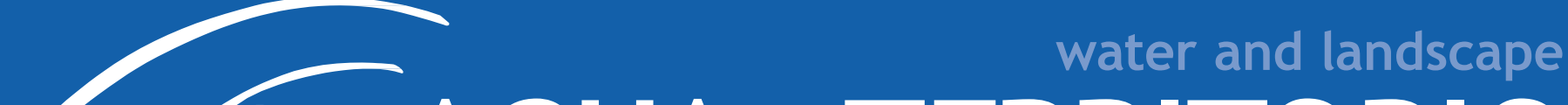 AGUA YTERRITORIO
}

\section{El activismo urbano frente a la crisis socioambiental y climática}

\author{
The urban activism against the socio-environmental and climatic crisis
}

\author{
Tommaso Gravante \\ Universidad Nacional Autónoma de México \\ Ciudad de México, México \\ t.gravante@gmail.com \\ https://orcid.org/0000-0003-1168-931X \\ Alice Poma \\ Universidad Nacional Autónoma de México \\ Ciudad de México, México \\ alicepoma@gmail.com \\ https://orcid.org/0000-0001-8755-6893
}

El 2019 ha sido el año del activismo climático y socioambiental. Millones de personas, con la novedosa participación de jóvenes y muy jóvenes, han inundado las calles de las ciudades de todo el planeta para expresar su preocupación por la falta de respuesta de los estados frente a la emergencia climática.

Junto a la masiva participación en los eventos de protesta y acciones organizadas por organizaciones transnacionales como Fridays For Future o Extinction Rebellion se observa también la difusión de agrupaciones y colectivos que defienden el agua, los bosques, los parques, etc. en las áreas metropolitanas, así como de otros que promueven un estilo de vida más respetuoso con la vida humana y no humana, a través de la producción y consumo de productos locales, artesanales, orgánicos, éticos y accesibles para la mayoría de la población.

Este dosier quiere visibilizar estas experiencias que, sumadas al ecologismo rural e indígena ya consolidado en América Latina (se piense en movimientos como Vía Campesina y en los numerosos conflictos socioambientales en defensa de la vida y los territorios en todo el continente), podrían representar una esperanza para enfrentar el actual colapso climático y ambiental, dando lugar a una nueva cultura proambiental.

Hemos considerado necesario dedicar un espacio al activismo proambiental urbano con la esperanza de estimular el estudio empírico de estas experiencias para así poder proporcionar herramientas teóricas, desde las ciencias sociales, a fin de comprender cómo puede cambiar - y está cambiando- nuestra relación con el planeta que habitamos y las demás especies, y cuáles son los desafíos y oportunidades que tenemos en frente.

Para poder entender esto es necesario aclarar qué es el activismo proambiental urbano.

El activismo es un concepto comúnmente empleado en Europa y Norteamérica, y puede ser voluntario o profesional, cuando las personas trabajan para organizaciones ambientalistas o ecologistas. El término activismo no es empleado en todos los países porque este concepto se usa comúnmente solo para identificar el activismo profesional y porque se prefiere el término militancia, más cercano a la tradición marxista. Desde esta perspectiva el activismo ha sido criticado porque se considera individualista o porque se asocia con la táctica de atraer la atención de los medios de comunicación a través de acciones muy mediáticas.

Stern et al. ${ }^{1}$ definen a los activistas (movement activists) como aquellas personas que están comprometidas en acciones públicas con el objetivo de influenciar el comportamiento del sistema político y de la población.

\footnotetext{
${ }^{1}$ Stern et al., 1999.
} 
Cuando el compromiso es reducido, los autores hablan de simpatizantes (supporters). El activismo, cuando es de base (en inglés se usa el término grassroots activism), presupone un rechazo a la forma de organización corporativista $\mathrm{y} / \mathrm{o}$ jerárquica, $\mathrm{y}$ ha sido muy exitoso para acercar a los movimientos sociales personas, sobretodo jóvenes, que a pesar de sentir la inquietud de querer hacer algo -en nuestro caso por el medio ambiente- no querían acercarse a organizaciones formales e instituciones, por no confiar en ellas. La reciente investigación sobre la nueva ola del movimiento climático en México ${ }^{2}$ ha demostrado, por ejemplo, que muchos de los jóvenes que se acercaron a Fridays For Future se identifican con el término activista, de la misma manera que se identifican como activistas personas adultas que desde hace años o décadas forman parte de grupos ecologistas. Por la misma encuesta, se puede apreciar que la mayoría de los entrevistados no confía en gobiernos y empresas para la resolución de la crisis climática. Por supuesto, la desconfianza en los actores institucionales y macroeconómicos es un elemento central para comprender el activismo socioambiental urbano, que busca promover el cambio social actuando desde el nivel local y desde abajo.

Aclarado el término activista, el segundo foco de atención es lo urbano. A pesar de que se hayan estudiado más los conflictos y movimientos socioambientales que surgen en zonas rurales, las ciudades alojan la mayor parte de la población del planeta, siendo al mismo tiempo víctimas y cómplices del deterioro ambiental.

Las ciudades juegan un papel importante en la mitigación del cambio climático porque "consumen una gran proporción de la energía producida globalmente - entre el 60 y el $80 \%$ - y son responsables de un porcentaje similar de las emisiones de $\mathrm{CO}_{2}$ del mundo"3. Como muestran varios estudios a nivel global ${ }^{4}$ será en las grandes ciudades donde se padecerán las mayores consecuencias del cambio climático, con impactos tan graves como la contaminación, las inundaciones, las olas de calor, las heladas, los regímenes de tormentas, la nubosidad y la radiación ${ }^{5}$. Además, en las zonas metropolitanas se cuenta con bosques urbanos y terrenos agrícolas que sufren los efectos del cambio del clima, como por ejemplo el aumento de los incendios forestales y la disminución de la producción agrícola local. Si a eso añadimos que la mitad de la población total del planeta vive ya en áreas urbanas —y se estima que ese porcentaje será del $75 \%$ antes de $2050^{6}$ — no sorprende que la comunidad científica internacional subraye la importancia de que las sociedades aceleren la adaptación al cambio climático, en particular en los países de ingresos medianos y bajos, donde el impacto puede causar mayores daños ${ }^{7}$.

El activismo urbano juega así un papel central para limitar la contaminación y el deterioro ambiental que genera el estilo de vida urbano y la expansión de las ciudades, y también para generar conciencia sobre el

\footnotetext{
${ }^{2}$ Gravante \& Poma, 2020.

${ }^{3}$ Velasco et al., 2014, 13.

${ }^{4}$ OECD, 2010. Aureli et al., 2016.

${ }^{5}$ Sánchez Rodríguez, 2013.

${ }^{6} \mathrm{UN}-\mathrm{Habitat}, 2011$.

${ }^{7}$ IPCC, 2012.
}

impacto del sistema de producción y consumo que caracteriza a las sociedades capitalistas.

Como escribe Amorós, las ciudades han sido planificadas para ser lugares destinados al movimiento de mercancías y dinero, más que de personas. Por eso "la lucha por liberar el espacio urbano será la nueva lucha de clases. Un programa radical ha de oponerse al desarrollismo y reclamar un retorno a la ciudad, es decir, al ágora, a la asamblea. (...) La ciudad ha de generar una atmósfera que al respirarla haga libres a sus habitantes" 8 .

Las ciudades serían lugares aún más contaminados e inhóspitos si no hubiera en ellas experiencias como las que promueven una nueva cultura vial donde el uso de la bicicleta o los peatones no son percibidos como un estorbo y se fomenta las infraestructuras que les permite poderse mover en total seguridad. Otras experiencias similares son los huertos urbanos, que proporcionan un lugar de encuentro social, educación ambiental y sustento alimentario, las pequeñas cooperativas de agricultores urbanos, que les permite producir y distribuir sus productos en los límites de las zonas metropolitanas. También destaca la existencia de colectivos, asociaciones civiles y movimientos que cuidan del territorio y denuncian las irregularidades en la gestión y manejo de los espacios verdes y bienes naturales que aún están presentes en estas áreas. Por supuesto todas estas experiencias tienen que lidiar con la falta de cultura proambiental en la mayoría de los países, y con los intereses económicos que presionan para que la vida humana y no humana solo sea una fuente de riqueza económica, desprestigiando - $-\mathrm{y}$ a veces despreciandoel valor (no económico) de la vida.

El futuro y la esperanza del planeta y de las ciudades mismas están así en todas las formas de resistencia al actual sistema capitalista, que como escribe Hochschild, no es solo un sistema económico, sino también cultural ${ }^{9}$. Es en las ciudades y en las áreas metropolitanas donde hay que reconstruir la relación con el medio ambiente y una cultura proambiental que permita defender los bienes naturales, oponerse a nuevas infraestructuras y al desarrollo urbanístico, y romper con el consumo de productos foráneos de baja calidad o con un mercado verde elitista que solo proporciona productos de calidad a precios inaccesibles para la gran mayoría de la población.

En el presente dosier hemos querido poner en evidencia el papel del activismo socioambiental urbano de base en la actual crisis climática que estamos viviendo a través de cinco textos que analizan problemáticas socioambientales diferentes, aunque todas ellas vinculadas de una forma $\mathrm{u}$ otra a la crisis climática.

Como texto de abertura hemos considerado oportuno insertar un trabajo de los coordinadores del monográfico en el cual se presenta de forma general el papel del activismo socioambiental de base dentro de la nueva ola del movimiento climático. Mientras en la literatura anglosajona encontramos diferentes obras que analizan el movimiento climático ${ }^{10}$ en la literatura en español aún escasean los trabajos analíticos sobre este movimiento. El objetivo del artículo es así introducir la literatura y el contexto en el que se está desarrollando la reciente

\footnotetext{
${ }^{8}$ Amorós, 2017, 110.

${ }^{9}$ Hochschild, 2008.

${ }^{10}$ Dietz \& Garrelts, 2014. Kleres \& Wettergren, 2017. Tokar, 2014.
} 
ola del movimiento climático. Utilizando los estudios comparados del proyecto internacional Protest for a future: Composition, mobilization and motives of the participants in Fridays For Future climate protests (2018-2020), los autores presentan tres elementos que caracterizan el activismo socioambiental y que consideran determinantes en el desarrollo de estrategias frente a la emergencia climática: la presencia de apegos al lugar local y global, la priorización de valores altruistas y biosféricos, y el carácter prefigurativo de estas prácticas.

Los artículos que siguen a este análisis global son estudios de casos que nos sitúan en una dimensión territorial delimitada, aunque las problemáticas socioambientales que las afectan son desafortunadamente globales. El texto de Jorge Regalado Santillán (Universidad de Guadalajara, México) y Rodrigo Rodríguez Guerrero (Instituto Tecnológico y de Estudios Superiores de Occidente, México) presenta las relaciones existentes entre la crisis socioambiental y climática y sus respuestas sociales en la zona metropolitana de Guadalajara (ZMG), en México. Los dos investigadores destacan el papel del activismo socioambiental de base en tres aspectos que se relación directamente con las estrategias necesarias para frenar las crisis social y climática: la producción agroecológica en contexto urbano y el consumo crítico, la defensa del territorio, de los bosques y los cuerpos de agua, y la reducción del uso de energías fósiles en la vida cotidiana. El panorama que destacan los autores se caracteriza por la existencia de alternativas que no dependen del modelo neoliberal y que demuestran que es posible, viable y deseable otra relación con la naturaleza que no sea la de la explotación.

Fernando Eurístides de la Cruz-Carrillo (Instituto Tecnológico y de Estudios Superiores de Monterrey, México) nos presenta los conflictos socioambientales ocurridos durante la última década (2008-2018) en el Área Metropolitana de Monterrey (AMM), estado de Nuevo León, en el norte de México. El autor analiza el repertorio de acción, las demandas y el proyecto político del Movimiento en Defensa de la Madre Tierra y de la Vida (MDMTV), actor colectivo contencioso que desde una postura conservacionista antagoniza ética y políticamente con empresas y gobiernos promotores de prácticas neoextractivas. La investigación nos muestra que estos conflictos socioambientales, más allá de la dicotomía antropocentrismo-biocentrismo, son el resultado de una disputa simbólica y ética entre quien considera la naturaleza como fuente de explotación - las empresas y los gobiernos - y la naturaleza vista como un bien común, cuya defensa da como resultado un proceso democrático de reapropiación colectiva.

Andrés Sierra Martínez, de El Colegio de México, nos ofrece otro enfoque de análisis sobre el activismo ambiental urbano: los impactos biográficos de la protesta. De hecho, el texto analiza los efectos de la protesta en la vida cotidiana de una organización vecinal, la Asamblea General de los Pueblos, Barrios, Colonias y Pedregales de Coyoacán (AGPC), que se constituye en el marco de un conflicto para la defensa del agua y el territorio al sur de la Ciudad de México. El autor, de forma muy bien articulada, destaca que el activismo ambiental urbano da paso a procesos de reconfiguración del territorio y la ciudadanía, detonando también procesos de construcción de alternativas orientadas a la producción democrática y sustentable de la ciudad. Como en otras experiencias, cuando las rutinas de la vida cotidiana se entrelazan con la protesta, esto da paso a la emergencia de nuevas experiencias de ciudadanía fundamentadas en las solidaridades, el apego al territorio y en la construcción de alternativas socioambientales en los territorios urbanos de por sí devastados.

El ultimo texto de Oliver Gabriel Hernández Lara y Aracely Rojas López, ambos de la Universidad Autónoma del Estado de México, presenta una investigación original en la cual se vincula el mundo del arte al proceso de subjetivación política. El artículo analiza la experiencia del Proyecto Sed - de la Compañía Nacional de Teatroque lleva a la escena las luchas en defensa del agua de cuatro comunidades en el Valle de México. La zona temporalmente autónoma del escenario logra, a través del elemento esencial del agua, articular sentidos comunes entre las luchas y en sujetos diversos como pueblos originarios, barrios populares, académicos, artistas y mujeres.

Los cinco textos que forman parte de este dosier presentan un esfuerzo novedoso y riguroso para dar luz sobre un fenómeno social tan poco estudiado como el activismo socioambiental de base en contexto urbano y su papel en la actual crisis climática y socioambiental. Los artículos presentan respuestas locales a problemáticas globales, desde diferentes enfoques. Felicitamos a los autores por el esfuerzo y la aportación, y estamos convencidos de que los lectores encontrarán informaciones y claves de lectura para comprender el nuevo reto global que la humanidad tendrá que afrontar: la salvaguardia de nuestro planeta, también en las ciudades.

\section{Bibliografía}

Amorós, M. 2017: Contra la nocividad. Anarquismo, antidesarrolismo, revolución. Guadalajara (México), Grietas Editores.

Aureli, A. et al. 2016: Agua, megaciudades y cambio climático. Retrato de 15 ciudades emblemáticas del mundo. París (Francia), Organización de las Naciones Unidas para la Educación, la Ciencia y la Cultura.

Dietz, M. \& Garrelts, H. 2014: Routledge Handbook of the Climate Change Movement. New York, Routledge.

Gravante, T. \& Poma, A. 2020: "Mexico", en de Moor, J., Uba, K., Wahlström, M., Wennerhag, M. \& De Vydt, M. (coords.): Protest for a future II: Composition, mobilization and motives of the participants in Fridays For Future climate protests on 20-27 September, 2019, in 19 cities around the world. Goteburgo, Swedish Research Council for Sustainable, 160-176. Development. Retrieved from: [https://osf.io/ asruw/]. DOI: 10.17605/OSF.IO/ASRUW.

Hochschild, A. R. 2008: La mercantilización de la vida intima. Apuntes de la casa y el trabajo. Buenos Aires, Katz editores.

Kleres, J. \& Wettergren, Å. 2017: "Fear, hope, anger, and guilt in climate activism", Social Movement Studies, 16 (5), 507-519. https://doi.org/10.1 080/14742837.2017.1344546.

IPCC, 2012: Managing the Risks of Extreme Events and Disasters to Advance Climate Change Adaptation. A Special Report of Working Groups I and II of the Intergovernmental Panel on Climate Change. Cambridge/New York (UK/USA), Cambridge University Press.

OECD, 2010: Cities and Climate Change. Paris (Francia), OECD Publishing. https://doi.org/10.1787/9789264091375-en

Sánchez Rodríguez, R. (ed.) 2013: Respuestas urbanas al cambio climático en América Latina. Santiago de Chile (Chile), CEPAL, Naciones Unidas.

Stern, P. C., Dietz, T., Abel, T., Guagnano, G. A. \& Kalof, L. 1999: "A ValueBelief-Norm Theory of Support for Social Movements: The Case of Environmentalism", Research in Human Ecology, 6 (2), 81-97. 
Tokar, B. 2014: Toward Climate Justice. Perspectives on the Climate Crisis and Social Change. Porsgrunn (Noruega), New Compass Press.

UN-Habitat 2011: Cities and Climate Change. Global Report on Human Settlements 2011. London/Washington DC (USA), Earthscan.
Velasco Rodríguez, G., Becerra Delgado, M. L., Vázquez Ramírez, I., Skwierinski Durán, A., Haro González, M. E. y Ortega Ramírez, M. 2014: Programa de Acción Climática de la Ciudad de México 20142020. Ciudad de México (México), Centro Mario Molina para Estudios Estratégicos sobre Energía y Medio Ambiente, A. C. 